\title{
An Idealized Prototype for Large-Scale Land-Atmosphere Coupling
}

\author{
Benjamin R. Lintner, ${ }^{*}$ Pierre Gentine, ${ }^{+}$Kirsten L. Findell, ${ }^{*}$ Fabio D’Andrea, ${ }^{@}$ \\ ADAM H. SOBEL, ${ }^{\&}, * *$ AND GUIDO D. SALVUCCI ${ }^{++}$ \\ * Department of Environmental Sciences, Rutgers, The State University of New Jersey, New Brunswick, New Jersey \\ ${ }^{+}$Department of Earth and Environmental Engineering, Columbia University, New York, New York \\ \# Geophysical Fluid Dynamics Laboratory, Princeton, New Jersey \\ @ Laboratoire de Météorologie Dynamique, École Normale Supérieure, Paris, France \\ \& Department of Applied Physics and Applied Mathematics, Columbia University, New York, New York \\ ** Lamont-Doherty Earth Observatory, Columbia University, Palisades, New York \\ ${ }^{++}$Department of Earth and Environment, Boston University, Boston, Massachusetts
}

(Manuscript received 21 September 2011, in final form 24 September 2012)

\begin{abstract}
A process-based, semianalytic prototype model for understanding large-scale land-atmosphere coupling is developed here. The metric for quantifying the coupling is the sensitivity of precipitation $P$ to soil moisture $W, \Delta P / \Delta W$. For a range of prototype parameters typical of conditions found over tropical or summertime continents, the sensitivity measure exhibits a broad minimum at intermediate soil moisture values. This minimum is attributed to a trade-off between evaporation (or evapotranspiration) $E$ and large-scale moisture convergence across the range of soil moisture states. For water-limited, low soil moisture conditions, $\Delta P / \Delta W$ is dominated by evaporative sensitivity $\Delta E / \Delta W$, reflecting high potential evaporation $E_{p}$ arising from relatively warm surface conditions and a moisture-deficient atmospheric column under dry surface conditions. By contrast, under high soil moisture (or energy limited) conditions, $\Delta E / \Delta W$ becomes slightly negative as $E_{p}$ decreases. However, because convergence and precipitation increase strongly with decreasing (drying) moisture advection, while soil moisture slowly saturates, $\Delta P / \Delta W$ is large. Variation of key parameters is shown to impact the magnitude of $\Delta P / \Delta W$, for example, increasing the time scale for deep convective adjustment lowers $\Delta P / \Delta W$ at a given $W$, especially on the moist side of the profile where convergence dominates. While the prototype's applicability for direct quantitative comparison with either observations or models is clearly limited, it nonetheless demonstrates how the complex interplay of surface turbulent and column radiative fluxes, deep convection, and horizontal and vertical moisture transport influences the coupling of the land surface and atmosphere that may be expected to occur in either more realistic models or observations.
\end{abstract}

\section{Introduction}

Although coupling between land surface and atmospheric processes is regarded as a significant modulator of climate system variability, isolating land-atmosphere coupling pathways in observations and models, such as potential feedbacks between soil moisture and precipitation, remains a significant challenge. Incomplete knowledge of the mechanisms of land-atmosphere interactions, not to mention how such mechanisms are ultimately represented in numerical weather prediction and climate models, limits forecast and predictive skill

Corresponding author address: Benjamin R. Lintner, Dept. of Environmental Sciences, Rutgers, The State University of New Jersey, 14 College Farm Rd., New Brunswick, NJ 08901-8551. E-mail: lintner@envsci.rutgers.edu across multiple time scales. Several factors contribute to the difficulty of assessing land-atmosphere interactions in observations and models. Some, such as limited data or coarse resolution, may be mitigated through increased sampling or finer resolution; others require more careful consideration. For example, the inherent heterogeneity of both the land surface and atmosphere, particularly at small spatial scales, may obscure the relationship between soil moisture and subsequent precipitation $(\mathrm{Li}$ and Avissar 1994; Pielke et al. 1998).

The feedback of soil moisture onto subsequent precipitation is postulated to depend on three necessary conditions (Koster and Suarez 2003). First, a sufficiently large soil moisture perturbation must be present. Second, evaporation (or evapotranspiration) must be sensitive to soil moisture, and, finally, precipitation must be sensitive to evaporation. Much of the contemporary 
research on land-atmosphere interactions has emphasized hotspots where soil moisture-precipitation coupling appears to be especially pronounced (Koster et al. 2004; D'Odorico and Porporato 2004), that is, where all three conditions are likely to be met. Model simulations show that such coupling hotspots often occur in transitional hydroclimatic regimes characterized by intermediate values of soil moisture and precipitation. An argument for hotspot occurrence under such conditions hinges on the trade-off between, on the one hand, the weak dependence of evapotranspiration on soil moisture in the saturation limit for humid, high rainfall conditions and, on the other hand, a tropospheric environment unfavorable to moist deep convection in too dry environments. Together, these conditions suggest maximization of the potential for land surface-atmosphere feedbacks between the driest and wettest extremes.

Of course, it is well known that models exhibit wide variation in how they simulate hotspots (Guo et al. 2006). Intermodel discrepancies may reflect differences in model parameterizations and the fidelity of model simulations in producing spatially consistent distributions of precipitation and soil moisture, although even within a given model, hotspots may not occur in all transition areas. Moreover, the simple argument for the existence of localized hotspots is qualitative rather than quantitative: while this argument provides guidance for anticipating conditions under which to anticipate hotspots, it does not offer quantitative predictions of hotspots and how these depend on hydroclimatic variables. Within the context of the three necessary ingredients for producing coupling, models may differ in the details of their soil moisture variability, their evaporation sensitivity to soil moisture, and/or the precipitation sensitivity to evaporation.

In the present study, we focus on the two sensitivity components in the soil moisture-precipitation feedback. To do this, we employ a steady-state, semianalytic prototype based on some simplifying assumptions for the atmospheric and land surface components of the climate system. In our view, closing the gap between our theoretical understanding of land-atmosphere coupling and its applicability to observed or simulated behavior requires use of idealized modeling. The prototype employed couples an idealized atmosphere derived from an intermediate-level complexity model to a simple bucket land surface model; it can be viewed as representing a $1 \mathrm{D}$ spatial transect across a hydroclimatic gradient between nonconvecting, dry surface conditions on one side and strongly convecting, saturated conditions on the other.

While our prototype is clearly a simplified representation of land-atmosphere coupling, it can be used to demonstrate how key atmospheric and land surface parameters may be anticipated to impact the coupling. Thus, one objective here is to emphasize how largescale conditions modulate the coupling. In contrast to many studies that adopt a de facto local view of landatmosphere coupling (i.e., relating the land surface conditions and atmosphere at a particular point or observation site), we explicitly address local as well as nonlocal effects, the latter reflecting (large scale) moisture advection and convergence. We further use our prototype to illustrate potential sources of discrepancy between models and observations and within the models themselves.

\section{Land-atmosphere coupling strength inferred from the GFDL AM2.1}

To motivate our study, we present results from version 2.1 of the Geophysical Fluid Dynamics Laboratory (GFDL) atmospheric model (AM2.1) GCM (GFDL Global Atmospheric Model Development Team 2004). Applying a methodology similar to Findell et al. (2011), we estimate metrics of tropical land surface-atmosphere coupling for December-February (DJF) from a 25-yr simulation forced by observed sea surface temperatures (Fig. 1). These metrics represent the sensitivity of 10-day mean daily precipitation to the 10-day mean 0900-1200 local time evaporative fraction (EF; Fig. 1a) and root-zone soil water (WTR; Fig. 1b), denoted as $\Delta P / \Delta \mathrm{EF}$ and $\Delta P / \Delta \mathrm{WTR}$, respectively. Here, EF is related to the partitioning of surface turbulent fluxes; that is, $\mathrm{EF}=E /(H+E)=1 /(1+B)$, where $B$ is the Bowen ratio, $B=H E^{-1}$, with $H$ the sensible heat flux ( $H$ and $E$ are in the same units; throughout this paper the units are millimeter per day). Fluctuations in EF may be driven by soil moisture fluctuations (Gentine et al. 2007, 2010, 2011).

Our consideration of 10-day means, as opposed to the daily means in Findell et al. (2011), is motivated by the assumptions of the prototype discussed below. Of course, the use of 10-day means introduces some ambiguity into the implied directionality of the relationship between $P$ and either EF or WTR, as soil moisture clearly responds to precipitation. However, in what follows, we are not directly interested in isolating the response of soil moisture (or surface conditions) to precipitation from the more subtle effect of surface conditions feeding back onto precipitation. Rather, we aim to assess the coupled behavior as a whole; that is, given an incremental change in the surface state, how much is precipitation changed?

The functional relationship between the mean $\Delta P / \Delta \mathrm{EF}$ curve and EF reflects increased sensitivity at higher 
a) $\Delta \mathbf{P} / \Delta \mathbf{E F}$

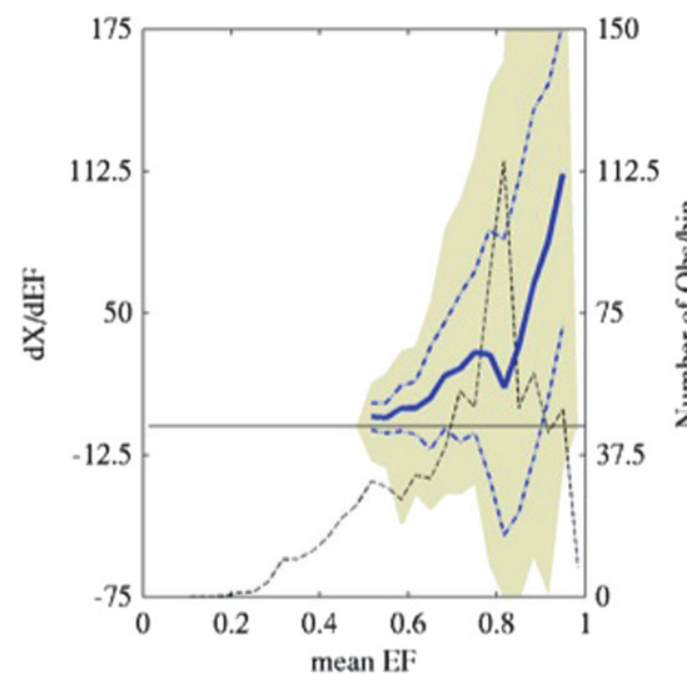

\section{b) $\Delta \mathbf{P} / \Delta \mathrm{WTR}$}

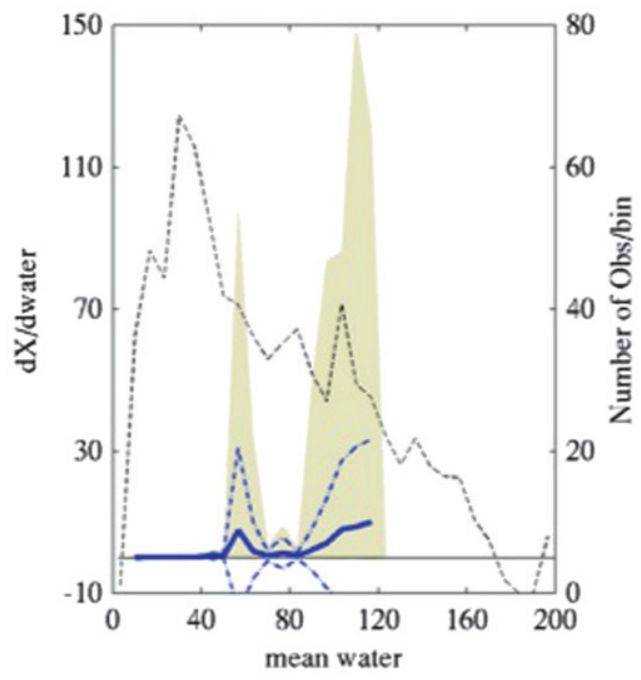

FIG. 1. Precipitation sensitivity as functions of (a) evaporative fraction $\Delta P / \Delta \mathrm{EF}$ and (b) soil water $\Delta P / \Delta \mathrm{WTR}$ as simulated by GFDL AM2.1. The sensitivities, expressed in units of millimeter per day per EF increment in (a) and millimeter per day per millimeter soil water in (b), are calculated as in Findell et al. (2011) using a binning procedure applied to 10-day averages of daily rainfall, including only those 10-day periods in the calculation for which precipitation exceeds a 1-mm threshold. The results shown are for 20 bootstrap samples generated from a 25 -yr model integration for all model land grid points between $30^{\circ} \mathrm{S}$ and $10^{\circ} \mathrm{N}$ for DJF. The dark blue lines represent means of the 20 bootstrap samples, the dashed blue lines represent the $\pm 1 \sigma$ level, and the tan shading corresponds to the range between the 5th and 95th percentiles. The dashed black lines (scaled along the right $y$ axes) are the pdfs of the DJF mean EF or WTR values.

EF, consistent with the findings of Findell et al. (2011). However, the large spread in the 5th-95th percentile values, estimated from 20 bootstrap samples of the original data, indicates substantial noise in the mean and even includes negative values of $\Delta P / \Delta E F$. For $\Delta P / \Delta \mathrm{EF}>0$, a positive excursion of morning EF [e.g., induced by increased soil moisture under constant radiative conditions at the surface; see Gentine et al. (2007)] would be expected to increase rainfall. It is of interest to note that the tropical grid points for which $\Delta P / \Delta \mathrm{EF}<0$ in AM2.1 are typically those for which convective precipitation is most intense (not shown). The reason for this negative sensitivity is not immediately clear.

Relating $\Delta P / \Delta$ WTR to WTR (Fig. 1b) shows the mean, 5th, and 95th percentile values to be positive everywhere. However, in contrast to the $\Delta P / \Delta E F$ versus EF relationship, $\Delta P / \Delta$ WTR versus WTR exhibits a distinct sensitivity minimum in the midrange of soil water values, both in its mean and in the 5th-95th percentile spread. In other words, the GFDL-simulated tropical land region precipitation increases less strongly in the midrange of soil water $(\sim 80 \mathrm{~mm})$ than it does at somewhat lower and higher values of WTR. For WTR $<40 \mathrm{~mm}$, where the probability distribution function (pdf) of WTR has its largest values, the sensitivity again decreases, which we suspect reflects conditions too dry for the GFDL model to trigger significant deep convection in DJF. Above $\sim 120 \mathrm{~mm}$, there are too few observations per WTR bin used to estimate the sensitivities, so $\Delta P / \Delta$ WTR is not calculated there. For now we note that a midrange sensitivity minimum appears at first to be at odds with the study of Koster et al. (2004), in which their soil moisture-precipitation coupling metric is argued to maximize at intermediate soil moisture values. However, the metrics considered here and in Koster et al. (2004) are substantively different, as their metric includes the variability in soil moisture. In the analysis below, we address the genesis of this minimum and discuss its potential implications for interpreting the soil moisture-precipitation feedback.

In the next section, we outline an analytic prototype for interpreting the precipitation sensitivity to soil moisture. The objective of this analysis is not to provide an encompassing quantitative explanation for the sensitivity but rather to develop a framework for diagnosing models and observations. The utility of this framework, in our view, is that it demonstrates, in a straightforward and physical manner, why the observed or simulated soil moisture-precipitation relationship may vary in magnitude across a well-defined hydroclimatic spatial gradient, or 
more generally, over distinct atmospheric and land surface states.

\section{Semianalytic prototype overview}

\section{a. Governing equations}

The prototype is distilled from a model of intermediatelevel complexity of the tropical atmosphere, the Quasiequilibrium Tropical Circulation Model 1 (QTCM1; Neelin and Zeng 2000; Zeng et al. 2000). Briefly, implementation of QTCM1 is guided by the postulate of quasi-equilibrium (QE), which provides a set of constraints for relating tropical deep convection, temperature, and circulation. Applying the QE constraints leads to a reduced vertical structure, which greatly diminishes the model's computational load and, for our purposes, facilitates the diagnosis and interpretation of the model.

The basis for our prototype is the simplest QTCM1, which comprises a single vertical temperature mode and barotropic and first baroclinic momentum modes; a single moisture mode is also invoked. The vertically averaged tropospheric temperature $T$ and moisture $q$ equations are given by

$$
\frac{\partial T}{\partial t}=-M_{s} \nabla_{H} \cdot \mathbf{v}+P+R_{\text {net }}+H-\mathbf{v}_{T} \cdot \nabla_{H} T \text { and }
$$

$$
\frac{\partial q}{\partial t}=M_{q} \nabla_{H} \cdot \mathbf{v}-P+E-\mathbf{v}_{q} \cdot \nabla_{H} q
$$

where $\nabla_{H}$ is the horizontal gradient operator, $R_{\text {net }}$ is the net column (top of the atmosphere minus surface) radiative heating, $M_{s}$ and $M_{q}$ are the dry static stability and moisture stratification (which are related to integrals over the vertical structures of temperature and moisture with momentum), $\nabla_{H} \cdot \mathbf{v}$ is signed positive for low-level convergence, and $\mathbf{v}_{T}$ and $\mathbf{v}_{q}$ are vertically averaged horizontal wind vectors weighted by the prescribed temperature and moisture vertical structures assumed in QTCM1. In the formulation of the vertically averaged equations, the terms in $P$ in (1) and (2) represent the net convective (condensational) heating and moistening, respectively; the negative sign in (2) indicates that $P$ is a tropospheric moisture sink. Moreover, all terms appearing in (1) and (2) are implicitly scaled to units of millimeters per day by absorbing constants such as specific heat capacity, latent heat of fusion, and $\Delta p / g$, where $\Delta p$ is the tropospheric pressure depth. A balanced surface flux constraint, neglecting ground surface heat flux, is also assumed:

$$
R_{\text {surf }}-E-H=0,
$$

where $R_{\text {surf }}$ is signed positive downward. During evaluation of (1)-(3), horizontal temperature gradients are assumed to be small (as in the tropics). Assuming flow in the zonal direction only, we consider moisture advection as in Sobel and Bellon (2009); that is, $u_{q}(\partial q / \partial x)=$ $-\tau_{\mathrm{adv}}^{-1}\left(q-q_{u}\right)$, where the $\tau_{\mathrm{adv}}$ is an advective time scale and $q_{u}$ is an upstream moisture value. We consider $\tau_{\mathrm{adv}}$ to be fixed and treat $q_{u}$ as an adjustable parameter, though it is also possible to adjust $\tau_{\text {adv }}$ for fixed $q_{u}$. The system of (1)-(3) is solved for $q, \nabla_{H} \cdot \mathbf{v}$, and surface temperature $T_{s}$ (see the appendix). These quantities depend parametrically on the evaporative efficiency $\beta=\beta(W)$. A closed-form, self-consistent solution can be obtained by invoking a steady soil moisture budget:

$$
P-E-Q=0,
$$

where $Q$ is the net runoff. For simplicity, $\beta(W)=W$ and $Q$ is represented as a simple power law, $Q=P W^{\alpha}$.

We note that Schaefli et al. (2012) have recently developed an analytic framework that shares some similarities with our prototype (e.g., consideration of advection along an "inflow" path into a region). One difference is that our prototype obtains moisture convergence as part of the solution rather than specifies it. The model of Schaefli et al. (2012) also contains a more detailed treatment of the land surface.

\section{b. Forcing and comparison with QTCM1}

In what follows, we consider the behavior of the prototype as the advection term is varied between 0 and a value such that moisture convergence precisely balances advection; for advection larger than this value, the prototype is in a nonconvecting regime with moisture convergence balancing advection and $P=0$ (see section 6). Solutions are obtained for a prescribed value of $T$.

Comparing the prototype solutions for $P, E$, and $W$ from the output of a QTCM1 simulation reveals broad agreement, particularly in the limit of small advection (Fig. 2). The QTCM1 results shown here are from a configuration comprising a tropical zonal strip with one land and one ocean region, as described in Lintner and Neelin (2009; cf. Fig. 2 of that paper for a schematic overview of the model configuration). For the parameter values chosen, this configuration produces a single convecting center over the midpoint of the land region. Because the horizontal moisture advection is monotonic in the zonal coordinate (i.e., its magnitude decreases inward toward the center of the convection zone), the large advection values to the right in Fig. 2, which here reflect drying advection that tends to suppress precipitation, 


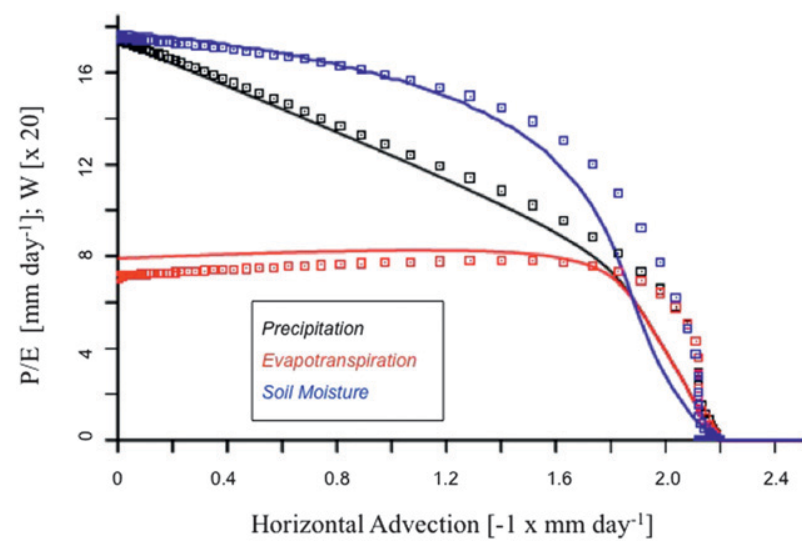

FIG. 2. Prototype solutions of precipitation $P$ (black line), evapotranspiration $E$ (red line), and soil moisture $W$ (blue line) as functions of the horizontal advection (here rescaled by a minus sign). Also shown are time-averaged values of $P, E$, and $W$ (squares) from a time-dependent QTCM1 simulation (see Lintner and Neelin 2009) configured in the same way as the prototype.

occur at the edge of the convection zone. In this region, the agreement is much less satisfactory given the time dependence of soil moisture, a point to which we return later. The offset between the prototype estimate of $E$ at relatively high soil moisture and $E$ from the full QTCM1 arises from the wind speed feedback on evaporation present in the latter; that is, horizontal wind speed decreases inward from the edge of the convection zone, which reduces the drag coefficient in the bulk formulation of potential evaporation.

\section{c. Prototype caveats}

We briefly remark here on a few limitations of the semianalytic model. Most significantly, the prototype lacks an explicit atmospheric boundary layer (ABL), owing to its use of single vertical temperature and moisture basis functions. The limited vertical degrees of freedom may be very important to manifestations of the soil moisture-precipitation relationship in our prototype; for example, Betts et al. (2007) note the importance of boundary layer clouds to land-atmosphere coupling. Moreover, some studies, including Findell et al. (2011), posit the feedback's operation in terms of convection triggering, which may ultimately depend on factors such as diurnal ABL growth with time-dependent solar heating. Our purpose is to emphasize how the coupling might be expected to impact the intensity of precipitation, assuming the state of the system can support deep convection (i.e., deep convection is already triggered). In related work (Gentine et al. 2013a,b), we are developing a coupled boundary layer-convection model that will explicitly address the role of triggering. Our analytic solutions also assume steady-state conditions, so that for a prescribed $T$, the prototype provides the fully adjusted behavior, similar to Entekhabi et al. (1992).

\section{Sensitivity of precipitation to soil moisture and its dependence on prototype parameters}

Using the solutions summarized in the appendix, we can estimate $\Delta P / \Delta W$ directly:

$$
\begin{aligned}
\frac{\Delta P}{\Delta W}= & {\left[P+\tau_{c}^{-1} q_{c}(T)\right]\left\{\Delta \ln \left[M_{s} M_{q}^{-1}\left(\epsilon_{T_{s}^{s}}^{T_{s}} G_{q}-\epsilon_{T_{s}}^{q} G_{T_{s}}\right)+\epsilon_{T_{s}^{s}}^{T_{s}} G_{T}-\epsilon_{T_{s}}^{T} G_{T_{s}}\right]\right.} \\
& \left.-\Delta \ln \left[M_{s} M_{q}^{-1}\left(\epsilon_{T_{s}}^{q} \epsilon_{q}^{T_{s}}-\epsilon_{q}^{q} \epsilon_{T_{s}}^{T_{s}}\right)+\epsilon_{T_{s}}^{T_{q}} \epsilon_{q_{s}}^{T_{s}}-\epsilon_{T_{s}^{s}}^{T_{s}} \epsilon_{q}^{T}\right]\right\} / \Delta W
\end{aligned}
$$

In writing (5), the moisture stratification has been taken as a constant, leading to a solution linear in $q$. However, a more general solution, with moisture stratification expressed as a linear function of $q$, is quadratic in moisture, although one root is nonphysical (i.e., $q<0$ ) for the parameter values considered here.

Despite the idealized formulation of the prototype, the behavior of (5) is nontrivial. We can speculate on some of the properties of $\Delta P / \Delta W$. First, the leading term in brackets on the rhs of (5) depends on the total precipitation and the threshold moisture value. This indicates that, in the limit as $P \rightarrow 0$, the sensitivity is nonzero: for our prototype, moisture advection balances moisture convergence in the limit $P \rightarrow 0$ (or $E \rightarrow 0$ ), so $P \rightarrow E$ and $\Delta P / \Delta W$ is dominated by the sensitivity of evapotranspiration $\Delta E / \Delta W$ in this limit. Also, in obtaining the prototype solution, we assume turbulent and radiative fluxes to be linearized about their mean values (which are dependent on large-scale $T$ ) and deviations from the mean are expressed in terms of $q$ and $T_{s}$. The mean values appear in the functions $G_{i}$, while deviations from the mean are reflected in the $\epsilon_{i}^{j}$ (as defined in the appendix). The $W$ dependence enters explicitly through $E$, so that each of the $G_{i}$ and $\epsilon_{i}^{j}$ associated with evapotranspiration depends on $\beta$. Thus, except for the terms $\epsilon_{T_{s}}^{T_{s}} G_{T}, \epsilon_{T_{s}}^{T} \epsilon_{q}^{T_{s}}$, and $\epsilon_{T_{s}}^{T_{s}} \epsilon_{q}^{T}$, all other terms in the arguments of the logarithms in (5) are quadratic in $W$.

Plotting $\Delta P / \Delta W$ as estimated from (5) as a function of $W$ (Fig. 3, black curve) indicates positive curvature across the entire range of soil moisture states, with 


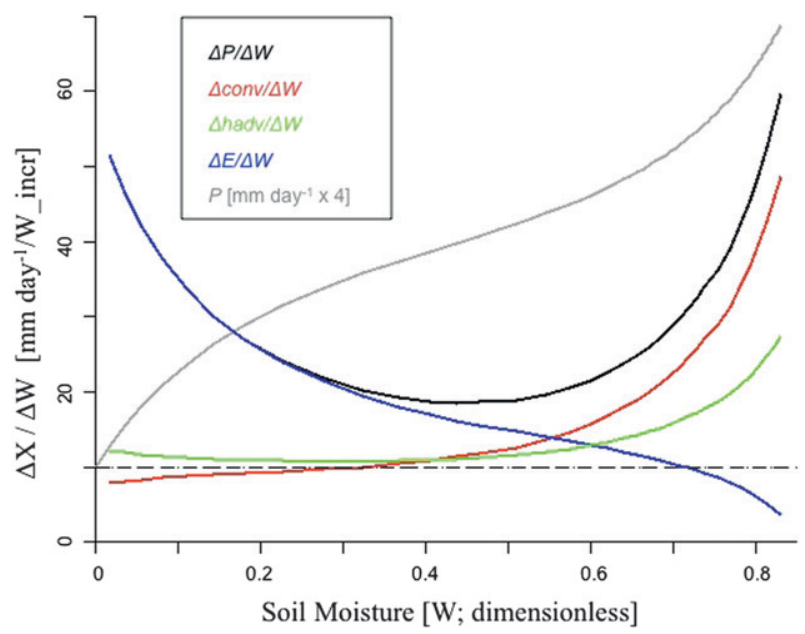

FIG. 3. Sensitivity of the steady-state prototype precipitation budget to soil moisture. Here, the sensitivity is estimated as the ratio of the incremental change in the budget term $X$ and soil moisture $W$ (i.e., $\Delta X / \Delta W)$. Also shown $\left(\mathrm{mm}^{-1 a y}{ }^{-1} \times 4\right)$ is the total precipitation as a function of $P$ (gray line).

a minimum sensitivity in the midrange of soil moisture values, consistent with the GFDL model results presented in Fig. 1b. In the next subsection, we address the source of this functional dependence. One notable difference with respect to the GFDL results is that large $\Delta P / \Delta W$ persists even down to $W=0$; we interpret this difference in terms of the steady-state nature of the prototype and the assumption that moisture is sufficiently high to trigger deep convection. We further point out that the behavior of $\Delta P / \Delta \mathrm{EF}$ plotted against $\mathrm{EF}$ (not shown) is also consistent with the GFDL AM2.1 results, namely $\Delta P / \Delta \mathrm{EF}$ increases with increasing $\mathrm{EF}$. The simple convective boundary layer model of De Ridder (1997) was found to produce qualitatively similar behavior, although that model was explicitly time dependent.

\section{a. Tropospheric moisture budget analysis}

To understand the variation in $\Delta P / \Delta W$ across the range of surface moisture conditions, it is instructive to examine the sensitivities of the other terms (evapotranspiration, moisture convergence, and horizontal advection) on the rhs of (2), the sum of which balances $P$ in steady state. For low soil moisture (water limited) conditions, $\Delta P / \Delta W$ largely mirrors the sensitivity of evapotranspiration to soil moisture $\Delta E / \Delta W$ (blue curve), as anticipated from the discussion above. With increasing $W, \Delta E / \Delta W$ decreases while the sensitivities of moisture convergence (red curve) and, to a lesser extent, horizontal advection (green curve) increase. At sufficiently large $W$, the sensitivity associated with moisture convergence dominates, with $\Delta P / \Delta W$ increasing, and, interestingly, $\Delta E / \Delta W$ becomes small and even slightly

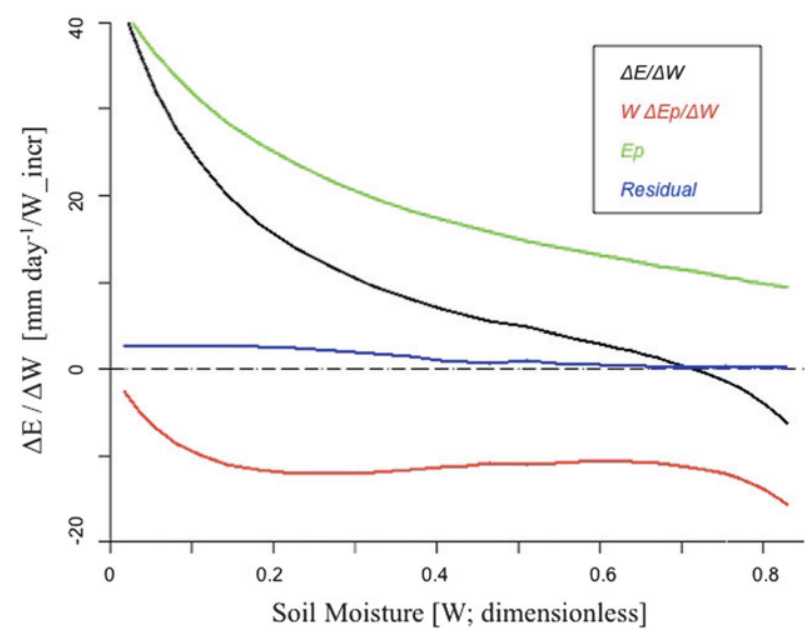

FIG. 4. Decomposition of evaporative sensitivity to soil moisture $\Delta E / \Delta W$. Component terms are discussed in the text.

negative, since the humidity deficit near the surface is reduced; that is, $E$ is reduced with increasing soil moisture through the reduction of potential evaporation induced by moisture convergence (see below). This behavior is of course anticipated from Fig. 2, which has $P$ increasing with decreasing (dry) moisture advection, with $E$ (slightly) decreasing. In addition, $W$ also increases, although it does so proportionally much less than $P$.

We can further consider the decomposition of $\Delta E / \Delta W$ itself:

$$
\frac{\Delta E}{\Delta W}=\frac{\Delta}{\Delta W}\left(\beta E_{p}\right)=E_{p}+W \frac{\Delta E_{p}}{\Delta W},
$$

which is depicted in Fig. 4. The first term on the rhs of (6), which is simply the potential evaporation, decreases with increasing soil moisture. This decrease can be understood by noting that, on the low soil moisture side, the surface is relatively warm while the overlying atmosphere is relatively dry, which corresponds to a relatively large gap between the saturation specific humidity (at the surface temperature) and the actual specific humidity (Bouchet 1963; Brutsaert and Stricker 1979). As the surface moistens, the equilibrium surface temperature decreases and the column moisture increases. In other words, as the Bowen ratio drops, latent heating increases (because of higher $W$ ) and sensible heating decreases: the shift toward latent heating and away from sensible heating results in $T_{s}$ decreasing and $q$ increasing. The second term on the rhs is negative over the range of $W$ since $E_{p}$ decreases monotonically with soil moisture.

While the physical pathway connecting soil moisture to precipitation through evaporation may be clear, how do we interpret the apparent linkage of soil moisture and 
moisture convergence? It is instructive here to consider the moisture convergence as the product of moisture and mass convergence; by combining (1) and (2) for the assumptions applied in the prototype, the mass convergence is just

$$
\nabla_{H} \cdot \mathbf{v}=M^{-1}\left(R_{\text {toa }}-u_{q} \frac{d q}{d x}\right)
$$

where $M=M_{s}-M_{q}$ is the gross moist stability. From this expression, the mass convergence is seen to comprise top-of-the-atmosphere (toa) net radiative heating and horizontal moisture advection. Thus, as drying advection (the second term on the rhs) decreases in magnitude, mass convergence increases. Combining (7) and (2) and computing a first-order perturbation gives

$$
\begin{aligned}
\delta P \approx & \delta E+\delta M_{q} \nabla_{H} \cdot \mathbf{v}+M_{q} M^{-1} \delta R_{\text {toa }} \\
& +M_{s} M^{-1} \delta\left(-u_{q} \frac{d q}{d x}\right) .
\end{aligned}
$$

We have seen that $\delta E$ is small in the limit of high $W$. Assuming that top-of-the-atmosphere radiative heating varies most strongly with the cloud-radiative feedback, the third term on the rhs can be expressed in terms of $\delta P$ itself. The second term can also be expressed in terms of $\delta P$ from the Betts-Miller formulation of precipitation [see (A5)]: under fixed $T, \delta P=\tau_{c}^{-1} \delta q$. Thus, from (8),

$$
\delta P \propto \delta\left(-u_{q} \frac{d q}{d x}\right) .
$$

This shows that precipitation responds more or less directly to advection for very wet surface conditions; the response is similar to what has been recently noted for a cloud-resolving model over the ocean (Wang and Sobel 2012). On the other hand, soil moisture itself varies relatively little as the surface approaches saturation. As a consequence, $\Delta P / \Delta W$ appears to be large. However, this should not be interpreted as indicating that small soil moisture increases drive large precipitation increases; rather horizontal moisture advection-the imposed external control parameter in these calculations-influences precipitation strongly but soil moisture only weakly, due at least in part to the saturation limit that constrains soil moisture but not precipitation.

\section{b. Sensitivity of $\Delta P / \Delta W$ to prototype parameters}

As an example of the dependence of $\Delta P / \Delta W$ on the prototype parameters, we examine what happens as the convective adjustment time scale $\tau_{c}$ is varied. Our consideration of this parameter is motivated by the fact that $\tau_{c}$, or its analogs of this time scale in other types of convection schemes, is currently poorly constrained: the range of potential values for this parameter is $2-16 \mathrm{~h}$ (Jackson et al. 2008). Given this spread, it is worthwhile to assess what impact varying this parameter may have on the prototype's precipitation sensitivity to soil moisture. We also note that this parameter is clearly on the "atmospheric side" of the coupled system; that is, it is independent of land surface formulations that are often viewed as the principal determinants of model discrepancy with respect to the soil moisture-precipitation feedback (Guo et al. 2006). It is therefore of interest to see how a change in such a parameter is reflected in the land-atmosphere coupling.

Increasing $\tau_{c}$ from its standard value of $2 \mathrm{~h}$ up to $16 \mathrm{~h}$ leads to a progressive lowering of the sensitivity at a given soil moisture value (Fig. 5a). However, the greatest impact from changing the convective adjustment time scale occurs at high soil moisture conditions. This is consistent with the strong relationship between convergence and precipitation on the moist side of the profile. Another aspect of increasing $\tau_{c}$ is the flattening of the region of minimum $\Delta P / \Delta W$; thus, while the sensitivity curves all exhibit the general U-shaped profile, the profiles widen at longer adjustment time scales.

Another highly uncertain process in current-generation models is the radiative impact of clouds (Bony et al. 2004; Solomon et al. 2007). In our prototype, cloud-radiative feedback is associated solely with precipitating deep convective conditions and encompasses surface and column radiative effects from both deep cumulonimbus and high anvil clouds. The radiative forcing associated with such clouds is expressed in terms of net surface and top-of-the-atmosphere feedback parameters $c_{\text {surf }}$ and $c_{\text {toa }}$ (see the appendix), for which respective baseline values of 0.18 and -0.08 (based on QTCM1) are assumed. Sobel et al. (2004) considered a range of values for $c_{\text {surf }}$ between 0 and 0.2 : halving $c_{\text {surf }}$ is found to increase $\Delta P / \Delta W$, but as with changes to $\tau_{c}$, the effect is largely confined to high soil moisture conditions (Fig. 5b). Based on the observed cancellation of shortwave and longwave radiative for tropical deep convective clouds (Kiehl 1994; Hartmann et al. 2001), it may in fact be reasonable to set the top-of-the-atmosphere forcing parameter $c_{\text {toa }}$ to zero. Changing the value of $c_{\text {toa }}$ has little impact on the sensitivity (not shown).

A final parameter we highlight briefly here pertains to the vertical structure of specific humidity. As noted above, the prototype is formulated in terms of vertically averaged moisture (and temperature). However, the bulk formula for $E_{p}$ depends on the surface moisture (parameter $b_{1 \mathrm{~s}}$ in the appendix). By varying the value assigned to $b_{1 \mathrm{~s}}$, the relative weighting of moisture can be shifted: assuming the vertical mean profile averaged over the 
a) Sensitivity to convective adjustment time

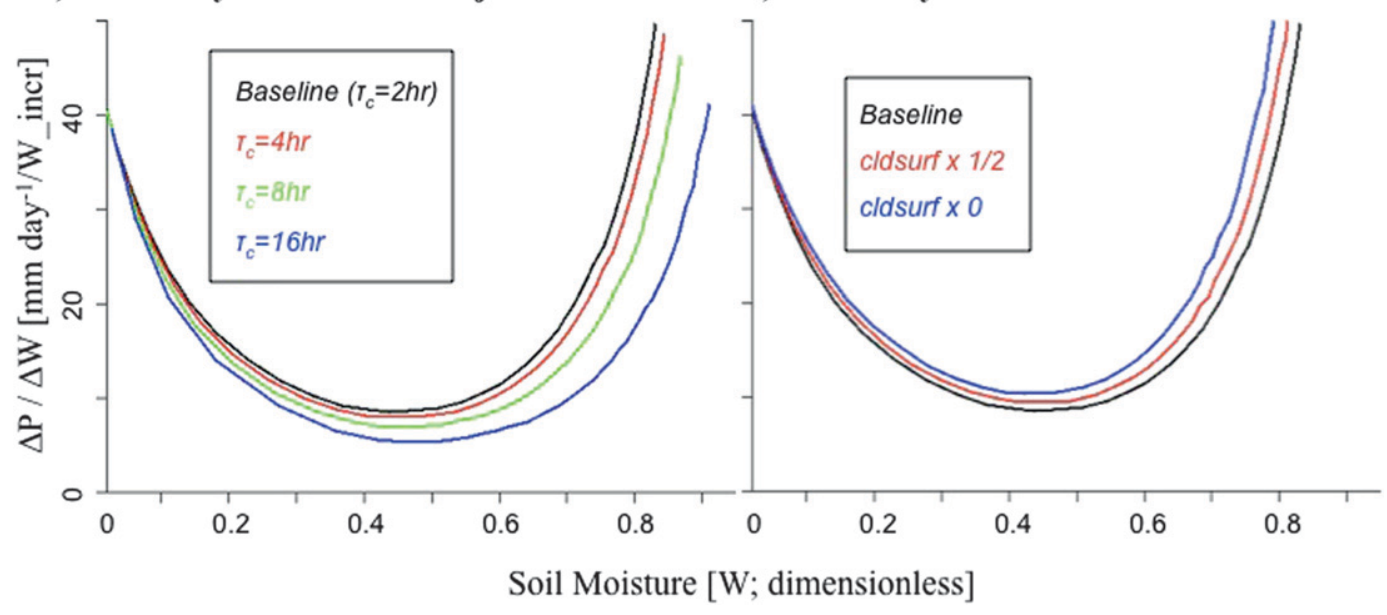

FIG. 5. Sensitivity of $\Delta P / \Delta W$ for varying (a) convective adjustment time scales $\tau_{c}$ and (b) the surface cloud-radiative feedback parameter. entire depth of the troposphere remains unchanged, increasing $b_{1 \mathrm{~s}}$ requires decreased weight in the layers above. We note that increasing $b_{1 \mathrm{~s}}$ decreases the sensitivity over the entire range of soil moisture, although the effect is small (not shown). This implies that, for two states with the same column water vapor but different boundary layer-free troposphere partitioning, the one with the moister boundary layer will exhibit (slightly) enhanced precipitation sensitivity to soil moisture. Whether similar sensitivity should hold in the presence of explicit boundary layer dynamics is uncertain.

\section{Sensitivity for disabling two-way coupling of evapotranspiration}

We have thus far not explicitly addressed what role the coupling of the land-atmosphere system plays in the precipitation sensitivity. In fact, precipitation sensitivity to soil moisture similar to our Fig. 3 has been previously demonstrated in an uncoupled stationary soil moisture balance model (Salvucci 2001). Using observed precipitation and other meteorological measurements to drive a soil-vegetation-atmosphere transfer (SVAT) model to estimate soil moisture and surface fluxes, Salvucci (2001) constructed conditional averages of $P, E$, and $Q$ on soil moisture and obtained a U-shaped profile of $\Delta P / \Delta W$.

The interpretation offered in Salvucci (2001) is that, under the assumption of stationarity, conditional averaging of $P$ on $W$ must reflect evapotranspiration at low soil moisture and runoff at high soil moisture, so consistent with (4), $\Delta P / \Delta W=\Delta E / \Delta W+\Delta Q / \Delta W$. Such results do not necessarily imply that coupling with the atmosphere is not playing a role: assuming the SVAT model simulations reflect what would be measured, the soil moisture and surface fluxes produced by the model would reflect land-atmosphere interaction. On the other hand, similar sensitivities have been obtained within an even simpler statistical model framework with evapotranspiration and runoff resembling those in the present study and forced by random perturbations to both $P$ and $E_{p}$. In this case, no land-atmosphere coupling is present.

To obtain some insight into the role of coupling in our prototype, we consider a configuration of the model for which the factors $\gamma$ and $b_{1 \mathrm{~s}}$ in the linearized expansion of evapotranspiration (A2) are set to zero. In this configuration, $E$ is simply dependent on soil moisture, while the effect of the atmospheric state on evapotranspiration is suppressed. Disabling the direct atmospheric impact on $E$ dramatically alters $\Delta P / \Delta W$ (Fig. 6, red curve) compared with Fig. 3: the sensitivity in this configuration increases monotonically over the range of soil moisture values. We suggest that the difference between this sensitivity curve and $\Delta P / \Delta W$ in Fig. 3 (dashed gray line) may be interpreted as the effect of the two-way coupling through evapotranspiration. At low $W$, this coupling enhances precipitation sensitivity to soil moisture, while at high $W$, it reduces it.

\section{Multiple equilibria and the bimodality of soil moisture probability distribution functions (pdfs)}

The results presented above have assumed a convectively triggered state; that is, the moisture balance is solved for moisture values such that $q \geq q_{c}(T)$. In fact, as discussed in Lintner and Neelin (2009), the prototype also supports a nonconvecting solution. Vertical mean moisture values for convecting and nonconvecting states 


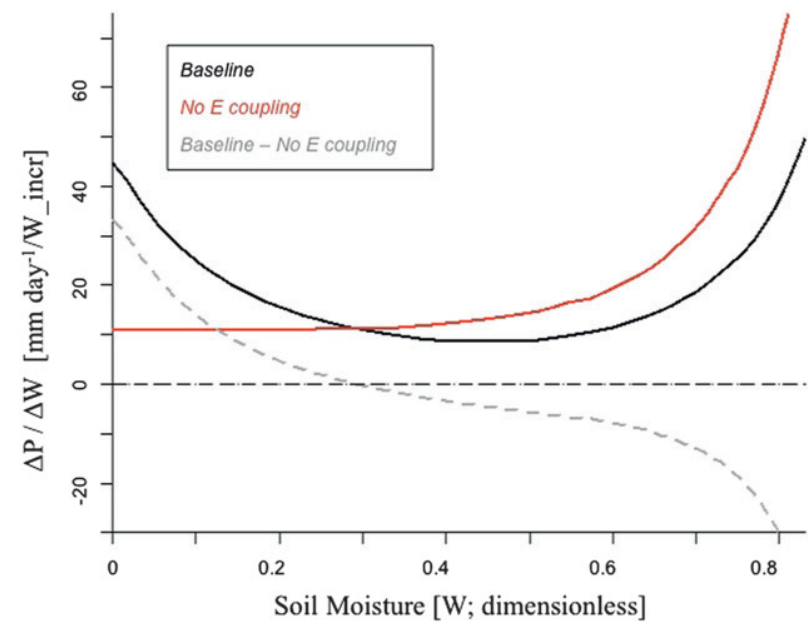

FIG. 6. Sensitivity of $\Delta P / \Delta W$ to decoupling $E$ from tropospheric moisture and surface temperature. Here, the no $E$-coupling configuration (red line) has $E=E(W)$, i.e., evapotranspiration as a function of soil moisture only. The difference between the baseline configuration and the no E-coupling configuration is also shown (dashed gray line).

are depicted in Fig. 7 as functions of the advective forcing. It can be seen that for a given value of moisture advection, the prototype admits both a low moisture/nonconvecting and high moisture/convecting state. Figure 7 also depicts the solution for $c_{\text {toa }}$ set to zero. Interestingly, the convecting solution for this case (red curve) is double valued for a small region near the convecting-nonconvecting transition value of moisture advection, which corresponds to convecting states with both low and high values of precipitation.

In prior work, convecting and nonconvecting solutions have been obtained in both single-column and cloud-resolving model simulations in weak temperature gradient mode for tropical oceans (Sobel et al. 2007; Sessions et al. 2010), with the principal difference being that for the steady-state land region, the nonconvecting latent heat flux is identically zero. Even for nonzero evaporation (and soil moisture) in the dry equilibrium over land, the surface temperature will change significantly with soil moisture, with the former increasing as the latter decreases, as under arid or semiarid conditions. Over oceans, surface conditions may not differ dramatically between the convecting and nonconvecting equilibria.

Under certain conditions, soil moisture pdfs have been shown to exhibit bimodality (D'Odorico and Porporato 2004; Teuling et al. 2005; D'Andrea et al. 2006), although the mechanisms for such bimodal behavior remain unclear. For example, it has been speculated that soil moisture bimodality represents a signature of the positive feedback between soil moisture and precipitation

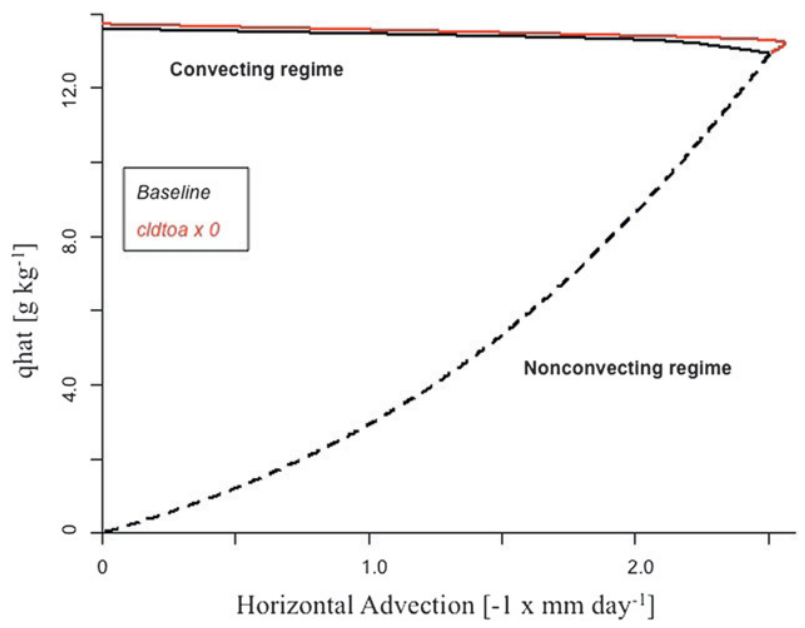

FIG. 7. Vertical mean moisture vs horizontal moisture advection for the nonconvecting solution of the prototype (dashed black line) and the convecting solution (solid black line). Also shown is the convecting solution for a configuration with toa cloud-radiative feedback set to zero (red line).

(see, e.g., D'Odorico and Porporato 2004). While the determination of such pdfs is obviously time dependent, the presence of convecting and nonconvecting solutions in our prototype could be envisioned to give rise to bimodal behavior in soil moisture, if one considers a succession of steady-state solutions. In this case, the shape of the pdf would depend on the relative frequency of occurrence of the nonconvecting, $W=0$ state and the convecting, nonzero soil moisture states.

\section{Summary and conclusions}

In this study, we develop an idealized, semianalytic prototype for understanding large-scale land-atmosphere coupling. Using this prototype, we show that the sensitivity of precipitation to soil moisture, defined as $\Delta P / \Delta W$, is characterized by a broad U-shaped profile, with the highest sensitivities at both extremes of $W$. From simple atmospheric moisture budget considerations, we illustrate how the shape of the $\Delta P / \Delta W$ profile reflects a trade-off between evapotranspiration, which dominates the sensitivity at low $W$, and moisture convergence, which dominates at high $W$. A key point here is that the large values of $\Delta P / \Delta W$ at high $W$ are attributable to the direct forcing of $P$ by moisture advection (the control parameter in the prototype) but with $W$ itself changing little.

In section 2, we noted the coupling metric of Koster et al. (2004), which is calculated from variances in simulations with and without interactive soil moisture. In contrast to Koster et al. (2004), we consider here only 


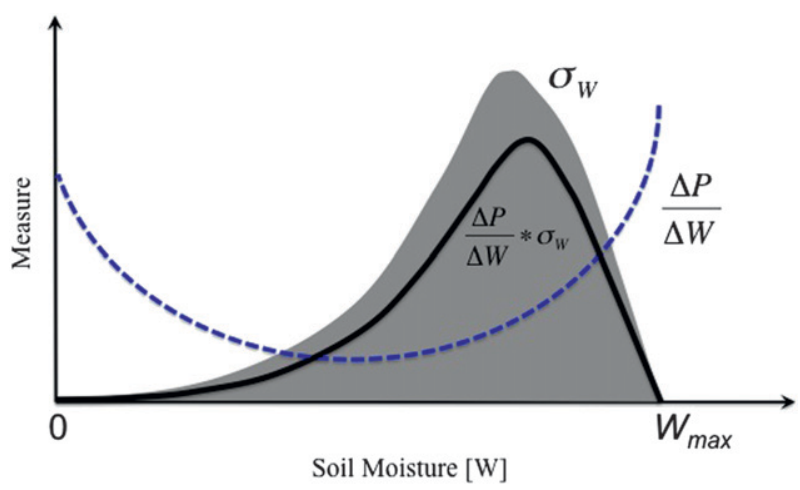

FIG. 8. Schematic illustration of precipitation sensitivity to soil moisture $\Delta P / \Delta W$ (dashed blue line), std dev of soil moisture $\sigma_{W}$ (gray shading), and their convolution $(\Delta P / \Delta W) * \sigma_{W}$ (solid black line).

the sensitivity of precipitation to a soil moisture change; that is, for a given soil moisture perturbation, we can estimate from $\Delta P / \Delta W$ how much precipitation is expected to change. This obviously bypasses how such soil moisture perturbations would occur in the first place. However, in broadly qualitative terms, we suggest that the convolution of our sensitivity with a measure of the soil moisture perturbations (e.g., the soil moisture standard deviation) should give something akin to the Koster et al. (2004) metric (see Fig. 8). For dimensionless soil moisture, which is bounded by 0 and 1 (or some $W_{\max }<1$ ), the distribution of soil moisture variance will approach zero at the endpoints, precisely where the sensitivity suggested by the prototype is largest.

Our study further suggests how $\Delta P / \Delta W$ may depend on parameters of interest in models, such as the convective adjustment time scale, cloud-radiative feedback strength, and vertical moisture distribution. These parameters are shown to impact the shape of $\Delta P / \Delta W$, which may help to explain (at least in a qualitative way) why models disagree in terms of where areas of strong or weak land-atmosphere coupling occur. For this reason, we suggest that examination of land-atmosphere coupling under systematic changes to parameters such as those used in convection schemes would be diagnostically useful.

Acknowledgments. The authors thank Alan K. Betts for discussion about this work and two anonymous reviewers for their comments. This work was supported by National Science Foundation (NSF) Grant AGS-1035968 and New Jersey Agricultural Experiment Station Hatch Grant NJ07102. AHS acknowledges support from NSF AGS-1008847.

\section{APPENDIX}

\section{Formulation of the Semianalytic Prototype}

To derive the prototype solution from (1) to (3), we first expand the turbulent and radiative fluxes as functions of $T, q$, and $T_{s}$ about flux offsets (0 subscripts):

$$
\begin{aligned}
& H=H_{0}+\epsilon_{H}\left(T_{s}-a_{1 \mathrm{~s}} T\right), \\
& E=\beta(W)\left[E p_{0}+\epsilon_{H}\left(\gamma T_{s}-b_{1 \mathrm{~s}} q\right)\right], \\
& R_{\text {surf }}=R_{\text {surf } 0}+\epsilon_{T_{s}}^{R_{\text {surf }}} T_{s}+\epsilon_{T}^{R_{\text {surf }}} T+\epsilon_{q}^{R_{\text {surf }}} q+c_{\text {surf }} P \text {, and } \\
& R_{\text {toa }}=R_{\text {toa } 0}+\epsilon_{T_{s}}^{R_{\text {toa }}} T_{s}+\epsilon_{T}^{R_{\text {toa }}} T+\epsilon_{q}^{R_{\text {toa }}} q+c_{\text {toa }} P .
\end{aligned}
$$

The $\epsilon$ coefficients represent the linear sensitivity of various fluxes to changes in $T, q$, and $T_{s}$. Precipitation (convective heating and drying) is formulated in terms of a Betts and Miller (1986) type relaxation scheme:

$$
P=\tau_{c}^{-1}\left[q-q_{c}(T)\right] .
$$

Here, $q_{c}(T)$ is a temperature-dependent moisture threshold, $\tau_{c}$ is the convective adjustment time scale, and it is necessary that $P \geq 0$. In addition, $c_{\text {surf }}$ and $c_{\text {toa }}$ are cloudradiative forcing coefficients associated with the presence of deep convective cloudiness and related anvil cirrus. Default values for the various parameters are summarized in Lintner and Neelin (2009). Solutions to (1)-(3) can then be written in the following format:

$$
q=\frac{\left[M_{s}\left(\epsilon_{T_{s}^{T}}^{T_{s}} G_{q}-\epsilon_{T_{s}}^{q} G_{q}\right)+M_{q}\left(\epsilon_{T_{s}}^{T_{s}} G_{T}-\epsilon_{T_{s}}^{T} G_{T_{s}}\right)\right]}{\left[M_{s}\left(\epsilon_{T_{s}}^{q} \epsilon_{q}^{T_{s}}-\epsilon_{T_{s}}^{T_{s}} \epsilon_{q}^{q}\right)+M_{q}\left(\epsilon_{T_{s}^{T}}^{T} \epsilon_{q}^{T_{s}}-\epsilon_{T_{s}^{s}}^{T_{s}} \epsilon_{q}^{T}\right)\right]},
$$

$$
T_{s}=-\epsilon_{T_{s}}^{T_{s}}\left(G_{T_{s}}+\epsilon_{q}^{T_{s}} q\right), \text { and }
$$

$$
\nabla_{H} \cdot \mathbf{v}=M_{s}^{-1}\left(G_{T}+\epsilon_{T_{s}}^{T} T_{s}+\epsilon_{q}^{T} q\right)
$$

Here, quantities of the form $\epsilon_{j}^{i}$ represent the net impact on variable $i\left(i=T, q, T_{s}\right)$ of those components of the linearized turbulent and radiative fluxes or the convective heating and drying rates depending on variable $j$. The quantities $G_{i}$ reflect the offset values as well and the $T$-dependent components of the fluxes.

\section{REFERENCES}

Betts, A. K., and M. J. Miller, 1986: A new convective adjustment scheme. Part II: Single column tests using GATE-wave, BOMEX, 
ATEX, and Arctic airmass data sets. Quart. J. Roy. Meteor. Soc., 112, 693-710.

— R. Desjardins, and D. Worth, 2007: Impact of agriculture, forest and cloud feedback on the surface energy balance in BOREAS. Agric. For. Meteor., 142, 156-169, doi:10.1016/ j.agrformet.2006.08.020.

Bony, S., J.-L. Dufresne, H. Le Treut, J.-J. Morcrette, and C. Senior, 2004: On dynamic and thermodynamic components of cloud changes. Climate Dyn., 22, 71-86, doi:10.1007/s00382-003-0369-6.

Bouchet, R. J., 1963: Evapotranspiration reelle et potentielle, signification climatique. Int. Assoc. Sci. Hydrol. Publ., 62, 134-142.

Brutsaert, W., and H. Stricker, 1979: An advection aridity approach to estimate regional evaporation. Water Resour. Res., 15, 443-450.

D'Andrea, F., A. Provenzale, R. Vautard, and N. De NobletDecoudré, 2006: Hot and cool summers: Multiple equilibria of the continental water cycle. Geophys. Res. Lett., 33, L24807, doi:10.1029/2006GL027972.

De Ridder, K., 1997: Land surface processes and the potential for convective precipitation. J. Geophys. Res., 102, 30 085-30 090

D'Odorico, P., and A. Porporato, 2004: Preferential states in soil moisture and climate dynamics. Proc. Natl. Acad. Sci. USA, 101, 8848-8851.

Entekhabi, D., I. Rodriguez-Iturbe, and R. L. Bras, 1992: Variability in large-scale water balance with land surface-atmosphere interaction. J. Climate, 5, 798-813.

Findell, K. L., P. Gentine, B. R. Lintner, and C. Kerr, 2011: Probability of afternoon precipitation in eastern United States and Mexico enhanced by high evaporation. Nat. Geosci., 4, 434-439, doi:10.1038/ngeo1174.

Gentine, P., D. Entekhabi, A. Chehbouni, G. Boulet, and B. Duchemin, 2007: Analysis of evaporative fraction diurnal behaviour. Agric. For. Meteor., 143, 13-29.

$\longrightarrow,-$, and J. Polcher, 2010: Spectral behaviour of a coupled land-surface and boundary-layer system. Bound.-Layer Meteor., 134, 157-180, doi:10.1007/s10546-009-9433-z.

—, _ and - 2011: The diurnal behavior of evaporative fraction in the soil-vegetation-atmospheric boundary layer. J. Hydrometeor., 12, 1530-1546.

_ A. K. Betts, K. L. Findell, B. R. Lintner, C. van Heerwaarden, A. Tzella, and F. D'Andrea, 2013a: A probabilistic-bulk model of coupled boundary layer and convection. Part I: Clear-sky case. J. Atmos. Sci., in press. bulk model of coupled boundary layer and convection. Part II Shallow convection case. J. Atmos. Sci., in press.

GFDL Global Atmospheric Model Development Team, 2004: The new GFDL global atmosphere and land model AM2-LM2: Evaluation with prescribed SST simulations. J. Climate, 17, 4641-4673.

Guo, Z., and the GLACE Team, 2006: GLACE: The Global LandAtmosphere Coupling Experiment. Part II: Analysis. J. Hydrometeor., 7, 611-625.

Hartmann, D. L., L. A. Moy, and Q. Fu, 2001: Tropical convection and the energy balance at the top of the atmosphere. J. Climate, 14, 4495-4511.
Jackson, C. S., M. K. Sen, G. Huerta, Y. Deng, and K. P. Bowman, 2008: Error reduction and convergence in climate prediction. J. Climate, 21, 6698-6709.

Kiehl, J. T., 1994: On the observed near cancellation between longwave and shortwave cloud forcing in tropical regions. J. Climate, 7, 559-565.

Koster, R. D., and M. J. Suarez, 2003: Impact of land surface initialization on seasonal precipitation and temperature prediction. J. Hydrometeor., 4, 408-423.

— between soil moisture and precipitation. Science, 305, 11381140.

Li, B., and R. Avissar, 1994: The impact of spatial variability of landsurface characteristics on land-surface heat fluxes. J. Climate, 7, 527-537.

Lintner, B. R., and J. D. Neelin, 2009: Soil moisture impacts on convective margins. J. Hydrometeor., 10, 1026-1039.

Neelin, J. D., and N. Zeng, 2000: A quasi-equilibrium tropical circulation model-Formulation. J. Atmos. Sci., 57, 1741-1766.

Pielke, R. A., R. Avissar, M. Raupach, A. J. Dolman, X. Zeng, and A. S. Denning, 1998: Interactions between the atmosphere and terrestrial ecosystems: Influence on weather and climate. Global Change Biol., 4, 461-475.

Salvucci, G. D., 2001: Estimating the moisture dependence of root zone water loss using conditionally averaged precipitation. Water Resour. Res., 37, 1357-1365.

Schaefli, B., R. J. van der Ent, R. Woods, and H. H. G. Savenije, 2012: An analytical model for soil-atmosphere feedback. Hydrol. Earth Syst. Sci., 16, 1863-1878, doi:10.5194/hess-16-1863-2012.

Sessions, S. L., S. Sugaya, D. J. Raymond, and A. H. Sobel, 2010: Multiple equilibria in a cloud-resolving model. J. Geophys. Res., 115, D12110, doi:10.1029/2009JD013376.

Sobel, A. H., and G. Bellon, 2009: The effect of imposed drying on parameterized deep convection. J. Atmos. Sci., 66, 2085-2096.

— C. S. Bretherton, H. Gildor, and M. E. Peters, 2004: Convection, cloud-radiative feedbacks and thermodynamic ocean coupling in simple models of the Walker circulation. Earth's Climate: The Ocean-Atmosphere Interaction, Geophys. Monogr., Vol. 147, Amer. Geophys. Union, 393-405.

-, G. Bellon, and J. Bacmeister, 2007: Multiple equilibria in a single-column model of the tropical atmosphere. Geophys. Res. Lett., 34, L22804, doi:10.1029/2007GL031320.

Solomon, S., D. Qin, M. Manning, M. Marquis, K. Averyt, M. M. B. Tignor, H. L. Miller Jr., and Z. Chen, Eds., 2007: Climate Change 2007: The Physical Science Basis. Cambridge University Press, 996 pp.

Teuling, A. J., R. Uijlenhoet, and P. A. Troch, 2005: Bimodality in warm season soil moisture observations. Geophys. Res. Lett., 32, L13402, doi:10.1029/2005GL023223.

Wang, S., and A. H. Sobel, 2012: Impact of imposed drying on deep convection in a cloud-resolving model. J. Geophys. Res., 117, D02112, doi:10.1029/2011JD016847.

Zeng, N., J. D. Neelin, and C. Chou, 2000: A quasi-equilibrium tropical circulation model-Implementation and simulation. J. Atmos. Sci., 57, 1767-1796. 